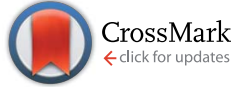

Cite this: RSC Adv., 2017, 7, 11021

Received 9th January 2017

Accepted 3rd February 2017

DOI: $10.1039 / \mathrm{c} 7 \mathrm{ra00346c}$

rsc.li/rsc-advances

\section{Surface charge switchable and core cross-linked polyurethane micelles as a reduction-triggered drug delivery system for cancer therapy $\dagger$}

\author{
Chang Liu, Yayuan Guan, Yuling Su, Lili Zhao, Fancui Meng, Yongchao Yao \\ and Jianbin Luo*
}

The extracellular stability versus intracellular drug release and the long blood stream circulation versus enhanced cell uptake are two dilemmas for micellar drug delivery systems. To resolve the above two problems, a novel core cross-linked polyurethane micelles with redox sensitive and $\mathrm{pH}$-responsive surface charge switchable properties were prepared and investigated as anti-cancer drug carriers. Firstly, amphiphilic multi-blocked polyurethane with pendant carboxyl groups and disulfides in the hard segment (PU-SS-COOH) was synthesized. Afterwards, 1,6-diaminohexane was connected onto some of the pendant carboxyl groups via dicyclohexylcarbodiimide (DCC) condensation, which resulted in polyurethane with pendant amine and carboxyl groups (PU-SS- $\mathrm{COOH}-\mathrm{NH}_{2}$ ). Then the PU-SS-COOH$\mathrm{NH}_{2}$ was assembled into micelles and the hexamethylene-1,6-diisocyanate (HDI) was added into the micelle solution. The hydrophobic diisocyanate penetrated into the hydrophobic core of PU micelles and reacted with amine groups, which resulted in core crosslinked PU micelles (PU-CCL). PTX and doxorubicin hydrochloride (DOX) were chosen as model hydrophobic drugs to evaluate the loading and redox-triggered release of the PU micelles. The drug release from the cross-linked polyurethane micelles (PU-CCL) demonstrated that the PU-CCL has superior particle stability compared with the uncross-linked polyurethane micelles in a phosphate buffer saline (PBS) solution without reducing agents, whereas the drug release rate is markedly accelerated with the addition of a reducing agent, glutathione (GSH). Notably, PU micelles with both amine and carboxyl groups, i.e. PU-SS-COOH- $\mathrm{NH}_{2}$ and PU-CCL micelles, showed $\mathrm{pH}$-responsive charge switchable properties. The in vitro cytotoxicity and cell uptake of the PTX (or DOX used to illustrate the cell uptake) loaded micelles was also assessed in HepG2 cells. It was found that the redox-sensitive polyurethane micelles can rapidly enter tumor cells. In vitro cytotoxicity studies demonstrated that the DOX-loaded PU-CCL display lower cytotoxicity in vitro compared to either free DOX or DOX-loaded uncrosslinked PU micelles. The cross-linked reduction responsive biodegradable micelles, having superior extracellular stability and providing rapid intracellular drug release, may hold great potential as a bio-triggered drug delivery system for cancer therapy.

\section{Introduction}

Recently, self-assembled polymer micelles based on multiblocked polyurethanes have been widely studied as anticancer drug carriers due to their highly tunable composition and good compatibility. ${ }^{1-5}$ After circulation in blood vessels, the nanoscale drug carriers accumulated passively at tumor sites owing to the enhanced permeation and retention (EPR) effect. $^{6-10}$ To achieve a better therapeutic effect, three more steps should be completed after the circulation and passive accumulation. The drug loaded carriers should penetrate into deep

College of Chemistry and Environmental Protection Engineering, Southwest University for Nationalities, 610041 Chengdu, China. E-mail: luojb1971@163.com

$\dagger$ Electronic supplementary information (ESI) available. See DOI: $10.1039 / \mathrm{c} 7 \mathrm{ra00346c}$ tumor tissues, be internalized by tumor cells and release drugs spontaneously in response to a specific stimulus specific to the intracellular micro-environment of the tumors, i.e. $\mathrm{pH}$ and redox. ${ }^{11-15}$ Redox-sensitive nanoscopic vehicles containing disulfide bonds which can be cleaved at millimolar concentrations of glutathione (GSH) are particularly interesting due to the great difference in the redox potential between the intracellular and extracellular compartments of tumor cells. ${ }^{16}$ As a consequence, polyurethanes with disulfide linkages in the backbone or side chains were extensively studied as efficient drug release systems. ${ }^{17,18}$

The rapid dilution of polymeric micelles after intravenous administration could cause the polymer concentration to fall below the critical micelle concentration (CMC), leading to premature drug release following injection at undesired sites. ${ }^{19-21}$ The early drug release not only lowers the local 
therapeutic efficacy, but also causes undesirable toxicity to normal organs. ${ }^{22}$ Crosslinked polymer micelles have been developed to improve the blood circulation stability of drug carriers and to decrease premature drug release. ${ }^{23-28}$ A PEG stealth strategy was widely used to prolong the circulation time of the nanoparticles in the blood by escaping the clearance of the nanoparticles by a reticuloendothelial system (RES). ${ }^{29-32}$ Neutral or negatively charged polymer micelles which avoid nonspecific binding during circulation have a longer circulation time than positively charged ones. ${ }^{33}$ However, the positively charged micelles have shown an enhanced cell uptake compared with negatively charged ones. ${ }^{34}$ To resolve the charge dilemma of drug carriers, surface charge switchable polymer micelles are highly anticipated. ${ }^{35,36}$ Polymer micelles that bear a negative charge in a neutral environment result in less protein adhesion and a prolonged circulation time in blood. When reaching tumor tissues there is an uptake by tumor cells when an acidic microenvironment is present; the negative surface charge of the polymer nanocarriers transforms to a positive one, leading to an enhanced cell uptake and lysosome escape. ${ }^{\mathbf{1 1}}$

Herein, novel core cross-linked polyurethane micelles with redox sensitive and $\mathrm{pH}$-responsive surface charge switchable properties were prepared and investigated as anti-cancer drug carriers. The process for the preparation of this kind of polyurethane micelles is shown in Scheme 1. Firstly, amphiphilic multi-blocked polyurethane with pendant carboxyl groups and disulfides in the hard segment (PU-SS-COOH) was synthesized by using poly(ethylene glycol) (PEG) and poly( $\varepsilon$-caprolactone) diol (PCL) as the soft-segment, and 2,2-bis(hydroxymethyl)propionic acid (DMPA) and cystamine dihydrochloride as the chain extenders. Afterwards, 1,6-diaminohexane was connected onto the partially pendant carboxyl groups via dicyclohexylcarbodiimide (DCC) condensation which resulted in polyurethane with pendant amine and carboxyl groups (PU-SS$\mathrm{COOH}-\mathrm{NH}_{2}$ ). Then the PU-SS-COOH- $\mathrm{NH}_{2}$ was assembled into micelles and the hexamethylene-1,6-diisocyanate (HDI) was

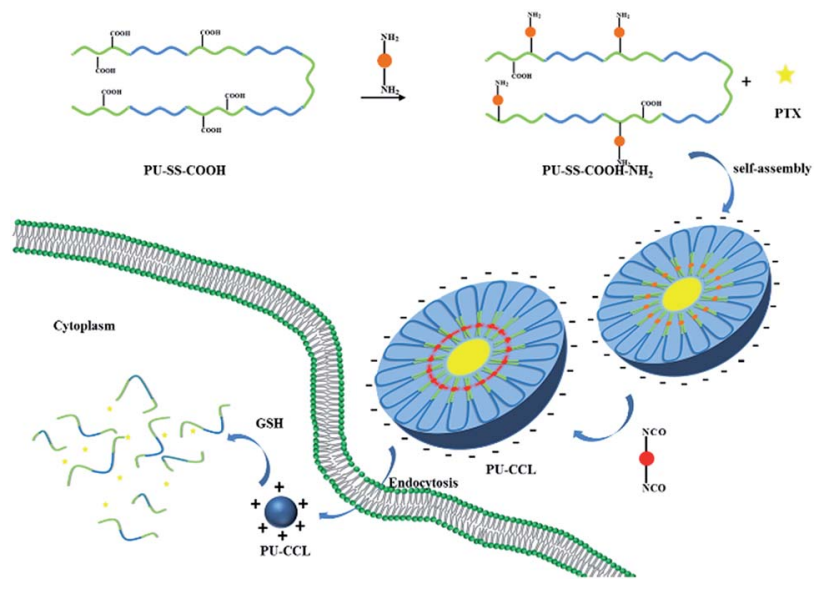

Scheme 1 Schematic illustration of the reduction sensitive polyurethane with carboxy groups (PU-SS-COOH), amine groups (PU-SS$\mathrm{COOH}-\mathrm{NH}_{2}$ ), the crosslinking process of $\mathrm{PU}-\mathrm{CCL}$ and their selfassembled PTX-loaded micelles as effective intracellular drug delivery nanocarriers. added into the micelle solution. The hydrophobic hexamethylene-1,6-diisocyanate penetrated into the hydrophobic core of PU micelles and reacted with amine groups, which resulted in core crosslinked PU micelles (PU-CCL). PTX and doxorubicin hydrochloride (DOX) were chosen as model hydrophobic drugs to evaluate the loading and redox-triggered release of the PU micelles. All the three kinds of PU micelles released their payloads in the presence of GSH, while PU micelles with both amine and carboxyl groups, i.e. PU-SS$\mathrm{COOH}-\mathrm{NH}_{2}$ and PU-CCL micelles, showed pH-responsive charge switchable properties. Furthermore, the stability of the core crosslinked PU micelles enhanced dramatically while the premature release decreased and the drug release of the PU-CCL slowed down compared with non-crosslinked micelles. The in vitro cytotoxicity and cell uptake of the PTX (or DOX, used to illustrate the cell uptake) loaded micelles were also assessed in HUVECs and HepG2 cells.

\section{Experimental}

\section{Materials}

Polyethylene glycol (PEG) $\left(M_{\mathrm{n}}=1000\right.$, Sunshine Biotechnology (Nanjing) Co., Ltd. China) and poly( $\varepsilon$-caprolactone)diol (PCL $M_{\mathrm{n}}$ $=2000$, Dow Chemical, USA) were dehydrated under reduced pressure at $100{ }^{\circ} \mathrm{C}$ for $2-3 \mathrm{~h}$ before use. $N, N$-Dimethylacetamide (DMAc) was dried over $\mathrm{CaH}_{2}$, vacuum distilled before use. Isophorone diisocyanate (IPDI), hexamethylene-1,6-diisocyanate (HDI) and stannous octoate $\left(\mathrm{Sn}(\mathrm{Oct})_{2}\right)$ were purchased from Aladdin Industrial Corporation, China, and used without further purification. Triethylamine (TEA, Aladdin Industrial Corporation, China) was distilled under vacuum. Cystamine dihydrochloride (Cys, Aladdin Industrial Corporation, China), 2,2-bis(hydroxymethyl)propionic acid (DMPA) and dicyclohexylcarbodiimide (DCC) were used as received. PTX (99.5\%) and doxorubicin hydrochloride (DOX, $-\mathrm{NH}_{3}{ }^{+} \mathrm{Cl}^{-}$salt form, >98\%) were obtained from AstaTech (Chengdu) Pharmaceutical Co. Ltd., China. Glutathione and sodium dodecyl sulfate (SDS) were purchased from Kelong Chemical Reagent Factory, China. The HUVEC cell lines were donated from the Shanghai Cell Bank of the Chinese Academy of Sciences. The HepG2 cell lines were obtained from the Wuhan Procell Life Science and Technology Co., Ltd. South American fetal bovine serum was obtained from CLARK Bioscience Co., Ltd. $0.25 \%$ of trypsin (+EDTA), RPMI/ medium, double resistance and PBS buffer were produced by Hyclone Co., Ltd. CCK8 was obtained from Biosharp Co., Ltd. DAPI was obtained from Roche Co., Ltd.

\section{Synthesis of PU-SS-COOH}

Polyurethane with pendant carboxyl groups and disulfide linkages on the main chain was synthesized from PEG, PCL, IPDI, Cys and DMPA by the traditional two step solution polymerization process. The feed ratios are listed in Table 1. In brief, anhydrous PEG and PCL-diol were dissolved in DMAc under a dry nitrogen atmosphere. IPDI was added into the DMAc solution of PCL and PEG and pre-polymerized in the presence of $0.1 \%$ stannous octoate $\left(\mathrm{Sn}(\mathrm{Oct})_{2}\right)$ at $90^{\circ} \mathrm{C}$ for $4 \mathrm{~h}$ under stirring. 


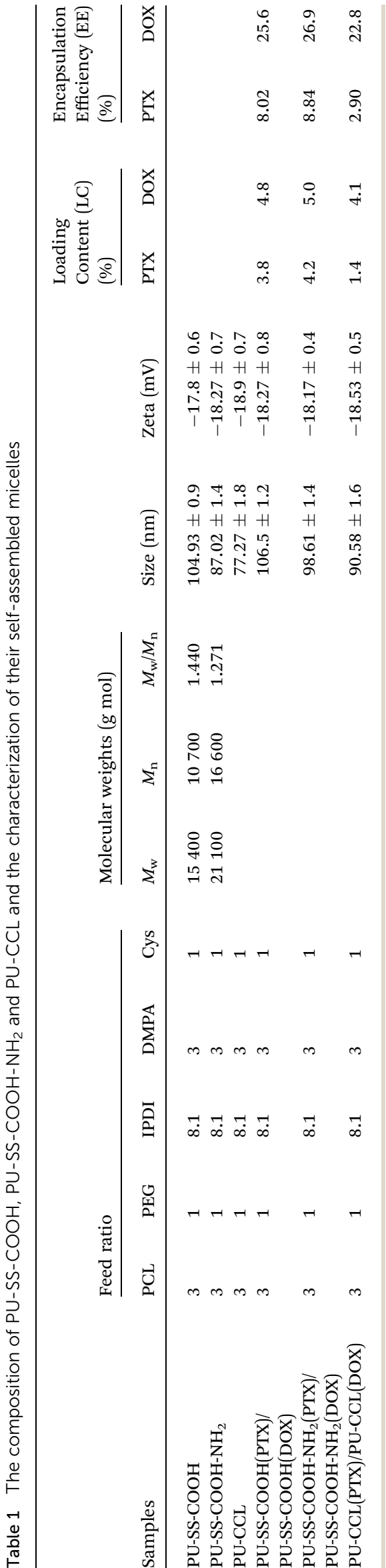

Afterwards, the prepolymer was chain extended by DMPA at $90{ }^{\circ} \mathrm{C}$ for another $4 \mathrm{~h}$. Then, the reaction solution was cooled to $0{ }^{\circ} \mathrm{C}$ by ice water and the chain extender Cys with the same amount of triethylamine (TEA, used to neutralize the hydrogen chloride of Cys) was added, and the mixture was kept at $0{ }^{\circ} \mathrm{C}$ for $1 \mathrm{~h}$ and at room temperature for $2 \mathrm{~h}$. Finally, the mixture was reacted at $90{ }^{\circ} \mathrm{C}$ for $4 \mathrm{~h}$ to complete the chain extending reaction. After that, the reaction mixture was cooled to room temperature. To remove the organic solvents and impurities, the crude product was subsequently poured into a water and methanol mixture with a volume ratio of $3: 1$. The precipitant was redissolved in DMAc and precipitated by a water and methanol mixture. The polymer was harvested and dried at $40{ }^{\circ} \mathrm{C}$ in the atmosphere for $5 \mathrm{~h}$ and at $60{ }^{\circ} \mathrm{C}$ in a reduced pressure for 2 days to get the final product.

\section{Synthesis of PU-SS-COOH-NH}

PU-SS-COOH (5 g) was dissolved in a mixture of DMF (15 ml) and THF $(20 \mathrm{ml})$ at room temperature with stirring. 1,6-Diaminohexane $(0.8 \mathrm{mmol})$ and 1-hydroxybenzotriazole (HOBT, 0.3 $\mathrm{mmol}$ ) was added and the reaction solution was placed into icesalt baths for $15 \mathrm{~min}$. Afterwards, dicyclohexylcarbodiimide (DCC, $2 \mathrm{mmol}$ ) was added under stirring and reacted for $18 \mathrm{~h}$ to complete the DCC condensation reaction. Polyurethane was purified using the same procedure as that to purify PU-SS$\mathrm{COOH}$. The final product was denoted as PU-SS-COOH-NH${ }_{2}$.

\section{Characterization of polyurethanes}

${ }^{1} \mathrm{H}$ NMR was recorded on an Agilent-NMR-vnmrs 400 (400 MHz) spectrometer using deuterated chloroform $\left(\mathrm{CDCl}_{3}-\mathrm{d} 6\right)$ as the solvent. The molecular weights and molecular weight distributions of PU-SS-COOH and PU-SS-COOH-NH $\mathrm{N}_{2}$ were determined by a Waters-1515 Gel Permeation Chromatogram. Tetrahydrofuran (THF) was used as the mobile phase at a flow rate of $1 \mathrm{ml}$ $\min ^{-1}$ at $40{ }^{\circ} \mathrm{C}$ and the molecular weights are reported relative to polystyrene (PS) standards.

\section{Preparation of polyurethane micelles}

A dialysis method was used to prepare the polyurethane micelles. Typically, polyurethane (PU-SS-COOH or PU-SSCOOH-NH $\mathrm{N}_{2}, 20 \mathrm{mg}$ ) was completely dissolved in $4 \mathrm{ml}$ DMF. Afterwards, the solution was added dropwise into $10 \mathrm{ml}$ distilled water under vigorous stirring. To prepare the PUCCL micelles, HDI (5 $\mathrm{mg} \mathrm{ml}^{-1}$ in DMF) was added slowly into the PU-SS-COOH- $\mathrm{NH}_{2}$ micelles in the presence of catalytic amounts of stannous octoate $\left(\mathrm{Sn}(\mathrm{Oct})_{2}\right)$ under intense stirring at room temperature. The reaction mixture was reacted under stirring at room temperature for 24 hours. Subsequently, the micelle solution was transferred to a dialysis bag (MWCO, $3.5 \mathrm{kDa}$ ) and dialyzed against distilled water for $72 \mathrm{~h}$ to eliminate the organic solvent at room temperature. The micelle solution was passed through a $0.45 \mu \mathrm{m}$ pore-sized syringe filter (Millipore, Carrigtwohill, Co. Cork, Ireland), and stored at $4{ }^{\circ} \mathrm{C}$. 


\section{Characterization of polyurethane micelles}

The critical micelle concentration (CMC) of the obtained polyurethanes in distilled water was determined by fluorescence spectroscopy using pyrene as a probe. The polyurethane concentration varied from $1.0 \times 10^{-5}$ to $0.2 \mathrm{mg} \mathrm{ml}^{-1}$ and the final pyrene concentration was fixed at $5.2 \times 10^{-6} \mathrm{M}$. The combined solution of pyrene and micelles was sonicated for $4 \mathrm{~h}$ in the dark before the fluorescence measurement. Fluorescence excitation spectra were recorded by a fluorometer (Varian Cary Eclipse Fluorescence Spectrophotometer, USA) at a wavelength range from 285 to $355 \mathrm{~nm}$, with the emission wavelength at $372 \mathrm{~nm}$ and slits at $5 \mathrm{~nm}$ for both excitation and emission. Size and zeta potentials of the nanoparticles in aqueous solution were measured with a Zetasizer analyzer (Malvern ZetasizerNano, Zen $3690+$ MPT2, Malvern, UK). The kinetic stability of the PU-SS$\mathrm{COOH}, \mathrm{PU}-\mathrm{SS}-\mathrm{COOH}-\mathrm{NH}_{2}$ and PU-CCL in DI water was studied in the presence of SDS acting as a destabilizing agent. The micelles $\left(1 \mathrm{mg} \mathrm{ml} \mathrm{m}^{-1}\right)$ were mixed with SDS aqueous solution $\left(20 \mathrm{mg} \mathrm{ml}^{-1}\right)$ in a $2: 1 \mathrm{v} / \mathrm{v}$ ratio and the scattered light intensity was monitored by DLS over a period of $48 \mathrm{~h} .^{28}$ The morphologies of PU-SS-COOH, PU-SS-COOH- $\mathrm{NH}_{2}$ and PU-CCL micelles were observed by transmission electron microscopy (TEM) (Tecnai $\mathrm{G}^{2}$ F20 transmission electron microscope, Royal Dutch Philips Electronics Ltd., Holland) at an accelerating voltage of $200 \mathrm{kV}$ with phosphotungstic acid $2 \%$ negative staining.

\section{Preparation of PTX-loaded micelles}

To prepare the PTX-loaded PU-SS-COOH and PU-SS-COOH-NH micelles, a clear solution consisting of the purified, dried PU (10 $\mathrm{mg}$ ) and PTX (5 mg) in DMF (1 ml) was added drop-wise to water $(10 \mathrm{ml})$. To prepare the PTX-loaded PU-CCL micelles, HDI $(5 \mathrm{mg}$ $\mathrm{ml}^{-1}$ in DMF) was added slowly into the PTX-loaded PU-SS-COOH$\mathrm{NH}_{2}$ micelles in the presence of a catalytic amount of stannous octoate $\left(\mathrm{Sn}(\mathrm{Oct})_{2}\right)$ under intense stirring at room temperature. The reaction mixture was reacted under stirring at room temperature for 24 hours. Subsequently, the micelle solution was transferred to the dialysis bag (MWCO, $3.5 \mathrm{kDa}$ ) and dialyzed against distilled water for $72 \mathrm{~h}$ to eliminate the organic solvent at room temperature. The excess of PTX was removed by a $0.45 \mu \mathrm{m}$ filter. The concentration of the drugs in the loaded micelles was evaluated using a high-performance liquid chromatography (HPLC) system (Waters Isocratic HPLC Pump, US) equipped with a reverse-phase C18 column $(4.6 \times 250 \mathrm{~mm}, 5 \mu \mathrm{m})$. An acetonitrile-water $(60 / 40 \mathrm{v} /$ v) mixture was used as the mobile phase and the flow rate was $1.0 \mathrm{ml} \mathrm{min}{ }^{-1}$. The UV adsorption of PTX that flowed out from the chromatographic column was recorded by a Waters $2489 \mathrm{UV} /$ Visible Detector at a wavelength of $227 \mathrm{~nm}$. The loading content $(\%)$ and encapsulation efficiency (\%) were calculated based on the equations below:

The loading content $(\mathrm{LC})(\%)=$ mass of drugs in micelles/total mass of loaded micelles $\times 100 \%$

Encapsulation efficiency $(\mathrm{EE})(\%)=$ mass of drugs in micelles/initial amount of feeding drugs $\times 100 \%$.

\section{Preparation of DOX-loaded micelles using dialysis method}

Water $(5 \mathrm{ml})$ was added drop-wise to a clear solution consisting of the purified, dried PU (10 mg), DOX ( $2 \mathrm{mg})$, and $\mathrm{Et}_{3} \mathrm{~N}$ (3 molar equivalents to DOX) in DMF $(2 \mathrm{ml})$. The resulting dispersion was dialyzed over water $(500 \mathrm{ml})$ for 2 days, yielding DOX-loaded micelles of PU at $1.0 \mathrm{mg} \mathrm{ml}^{-1}$. The excess DOX was removed by a $0.45 \mu \mathrm{m}$ filter. The concentration of the drugs in the loaded micelles was evaluated using a UV-vis spectrophotometer (UnicamUA500, Thermo Electron Corporation). The UV adsorption of DOX was recorded at a wavelength of $482 \mathrm{~nm}$. The loading content (\%) and encapsulation efficiency (\%) were calculated based on the equations below:

The loading content (LC) $(\%)=$

mass of drugs in micelles/total mass of loaded micelles $\times 100 \%$

Encapsulation efficiency (EE) $(\%)=$

mass of drugs in micelles/initial amount of feeding drugs $\times 100 \%$.

\section{In vitro release of PTX}

In vitro drug release from the drug-loaded micelles was performed using a dialysis method. In brief, $2.5 \mathrm{ml}$ of the drug loaded micelle solution (PU-SS-COOH, PU-SS-COOH-NH CCL) was added to a dialysis bag (MWCO: 8000) and immersed in $25 \mathrm{ml}$ of PBS (0.01 M, pH 7.4) containing $0.1 \mathrm{M}$ sodium salicylate with or without $10 \mathrm{mM}$ GSH. The dialysis system was kept at $37{ }^{\circ} \mathrm{C}$ in a thermostatic incubator with a shaking speed of $110 \mathrm{rpm}$. Samples were taken out and replaced with the same volume of fresh medium at the desired time intervals. The concentration of PTX released from the drug loaded micelles was analyzed using a HPLC.

\section{Cell culture}

Human liver cell line HepG2 cells were cultured in RPMI 1640 medium supplemented with $2 \mathrm{mM}$ L-glutamine, $100 \mathrm{U} \mathrm{ml}^{-1}$ penicillin and $10 \%$ fetal bovine serum (FBS) (FBS, CLARK, UT) at $37^{\circ} \mathrm{C}$ in a humidified atmosphere containing $5 \% \mathrm{CO}_{2}$ (Sanyo Incubator, MCO-18AIC, Japan).

HUVECs were maintained in Dulbecco's modified Eagle's medium (DMEM, Gibco Life, Grand Island, NY, USA) supplemented with $10 \%(\mathrm{v} / \mathrm{v})$ fetal bovine serum (FBS, CLARK, USA), $2 \mathrm{mM}$ L-glutamine and $1 \%(\mathrm{v} / \mathrm{v})$ antibiotics mixture $(10000 \mathrm{U}$ of penicillin and $10 \mathrm{mg}$ of streptomycin) (Hyclone). The cells were incubated in a humidified atmosphere of $5 \% \mathrm{CO}_{2}$ at $37{ }^{\circ} \mathrm{C}$ (Sanyo Incubator, MCO-18AIC, Japan).

\section{Cellular uptake and intracellular release of payloads}

Confocal laser scanning microscopy (CLSM) was employed to examine the cellular uptake and intracellular release behaviors of polyurethane micelles. The DOX-loaded micelles were incubated with HepG2 cells for $1 \mathrm{~h}$ at $37{ }^{\circ} \mathrm{C}$. After removal of the medium, the cells were washed three times with cold PBS, fixed with $1 \mathrm{ml}$ of $4 \%$ paraformaldehyde for $30 \mathrm{~min}$ at $4{ }^{\circ} \mathrm{C}$, and stained with 2-(4-amidinophenyl)-6-indolecarbamidine dihydrochloride (DAPI, Roche) for $10 \mathrm{~min}$. Finally, the slides were 
mounted with $10 \%$ glycerol solution and observed by a LeicaTCS SP8 (Leica Microscopy Systems Ltd., Germany).

\section{Cell viability assay}

To evaluate the antitumor activity of PTX-loaded polyurethane micelles and the cytocompatibility of the drug-free micelles, HepG2 cells and HUVECs were seeded in 96-well plates at $4 \times$ $10^{3}$ cells per well and incubated for $24 \mathrm{~h} \cdot{ }^{37}$ The culture medium was removed and replaced with $100 \mathrm{ml}$ medium containing various concentrations of micelle solutions for another $24 \mathrm{~h}$ of incubation. Then, $10 \mu \mathrm{m}$ of Cell Counting Kit-8 (CCK-8) solution (100T ml ${ }^{-1}$, Biosharp Co., Ltd.) was added to each well. After incubating the cells for $4 \mathrm{~h}$, the absorbance was measured at a wavelength of $450 \mathrm{~nm}$. The cell viability was normalized to that of cells cultured in the full culture media. The dose-effect curves were plotted and the median inhibitory concentration $\left(\mathrm{IC}_{50}\right)$ was determined using the software IBM SPSS Statistics (SPPS, Inc., USA).

\section{Results and discussion}

\section{Synthesis and characterization of polyurethanes}

The synthesis processes of the reduction-sensitive biodegradable multi-block polyurethanes are listed in Scheme 2. For PUSS-COOH, the feed ratio of PCL to PEG was $3: 1$ as listed in Table 1 . As we have reported previously, ${ }^{18}$ the disulfide bonds and carboxyl bonds were mainly located between the hydrophobic PCL segments, which resulted in the core location of carboxyl groups in the self-assembled micelles of PU-SS-COOH and PU-SS-COOH-NH$H_{2}$. Polyurethane with pendant primary

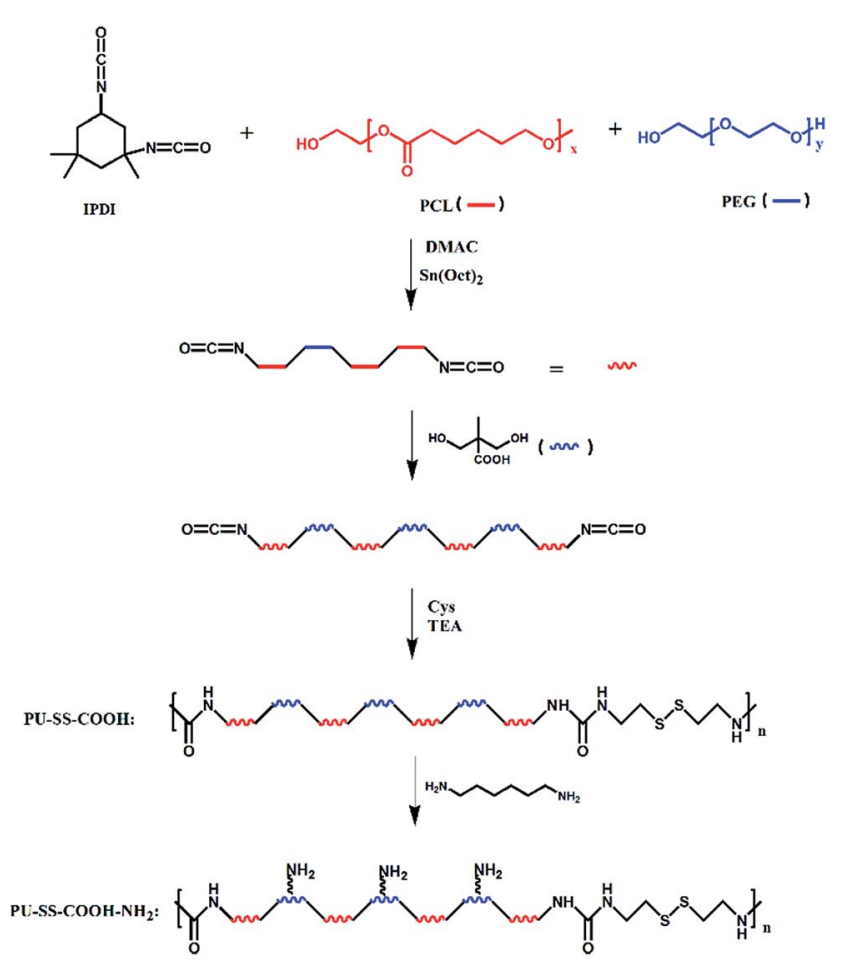

Scheme 2 Synthesis route of PU-SS-COOH and PU-SS-COOH-NH 2 . amine groups and carboxyl groups (PU-SS-COOH- $\mathrm{NH}_{2}$ ) were prepared by grafting 1,6-diaminohexane onto carboxyl groups using the DCC condensation reaction, as shown in Scheme 2.

The unimodal GPC curves of purified PU-SS-COOH and PUSS-COOH-NH $\mathrm{H}_{2}$ (Fig. 1) confirmed a successful polymerization. Notably, as is shown in Fig. 1, the retention time of PU-SS$\mathrm{COOH}-\mathrm{NH}_{2}$ is a little shorter than that of PU-SS-COOH, suggesting the successful grafting of 1,6-diaminohexane. The average molecular weight and polydispersity of PU-SS-COOH and PU-SS-COOH- $\mathrm{NH}_{2}$ are listed in Table 1. As shown in Table 1, PU-SS-COOH had $M_{\mathrm{n}}=10749 \mathrm{~g} \mathrm{~mol}^{-1}$ with $M_{\mathrm{w}} / M_{\mathrm{n}}=1.44$, while the $M_{\mathrm{n}}$ of PU-SS-COOH-NH $\mathrm{N}_{2}$ was $16615 \mathrm{~g} \mathrm{~mol}^{-1}$ with $M_{\mathrm{w}} /$ $M_{\mathrm{n}}=1.27$.

The representative ${ }^{1} \mathrm{H}$ NMR spectra of PU-SS-COOH and PUSS-COOH- $-\mathrm{NH}_{2}$ and the assignment of the peaks are presented in Fig. 2. The resonance peak centered at $3.62 \mathrm{ppm}$ (peak g) was assigned to the protons on the PEG units while the peaks at $4.03 \mathrm{ppm}$ (peak f), $2.28 \mathrm{ppm}$ (peak b), $1.62 \mathrm{ppm}$ (peaks c and e) and $1.36 \mathrm{ppm}$ (peak d) are attributed to the protons of the PCL units. The resonances at $1.22 \mathrm{ppm}$ (peak j), $1.02 \mathrm{ppm}$ (peak i) and $0.9 \mathrm{ppm}$ (peak h) are ascribed to the methylene and methyl protons of the IPDI units in the products. The peaks at $2.89 \mathrm{ppm}$ (peak a) are attributed to the methylene protons of the Cys units, indicating the presence of the Cys units in the resulting polyurethanes. The resonances at $4.24 \mathrm{ppm}$ (peak k) and $1.35 \mathrm{ppm}$ (peak l) are ascribed to the methylene and methyl protons of the DMPA units in the products, indicating the presence of the carboxyl groups in the PU-SS-COOH.

The resonances at $3.16 \mathrm{ppm}$ (peak p), $1.88 \mathrm{ppm}$ (peak m), $1.62 \mathrm{ppm}$ (peak n) and $1.35 \mathrm{ppm}$ (peak o) are ascribed to the amine and methyl protons of the 1,6-diaminohexane units in PU-SS-COOH- $\mathrm{NH}_{2}$, suggesting the successful attachment of 1,6diaminohexane onto the carboxyl groups of PU-SS-COOH and the presence of the primary amine groups in PU-SS-COOH- $\mathrm{NH}_{2}$. Based on the peak area ratio of peak $\mathrm{k}$ to peak $\mathrm{p}$, which correspond to the methane protons of DMPA and 1,6-diaminohexane, respectively, about $63 \%$ (molar ratio) of the carboxyl groups were grafted by 1,6-diaminohexane. Therefore, the molar ratio of primary amine to the carboxyl groups is 63/37 in PU-SS$\mathrm{COOH}-\mathrm{NH}_{2}$. The presence of both primary amine and carboxyl groups in the PU-SS-COOH- $\mathrm{NH}_{2}$ results in the charge switchable properties of PU-SS-COOH-NH${ }_{2}$ based micelles.

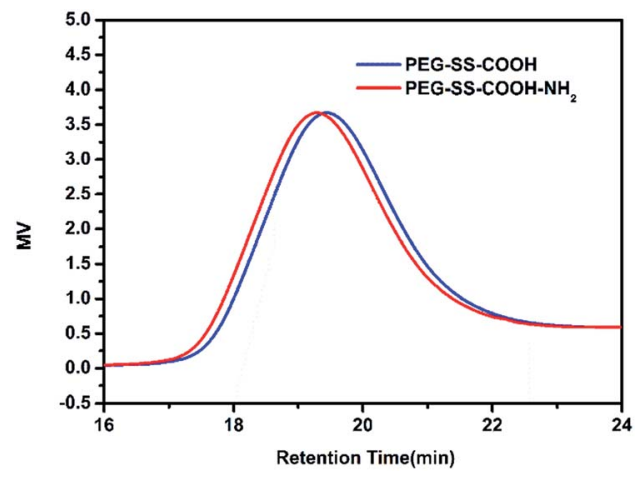

Fig. 1 GPC curves of PU-SS-COOH and PU-SS-COOH-NH 2 . 

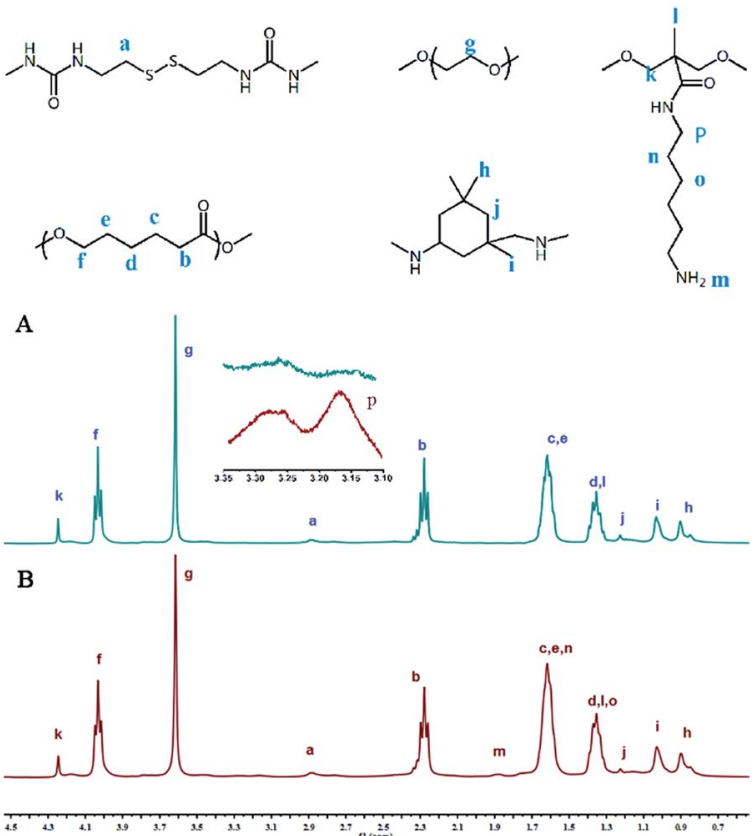

Fig. $2{ }^{1} \mathrm{H}$ NMR spectra of PU-SS-COOH (A) and PU-SS-COOH-NH $\mathrm{N}_{2}$ (B) in $\mathrm{CDCl}_{3}-\mathrm{d} 6$.

\section{Characterization of PU micelles}

The amphiphilic multi-blocked polyurethanes with redox responsive properties self-assembled in aqueous solution into micelles having a hydrophobic PCL core and a hydrophilic PEG shell. The core-shell structure of the polyurethane micelles was confirmed from fluorescence measurements using pyrene as a probe (ESI: Fig. S1A and $\mathrm{B} \dagger$ ).

The critical micellar concentration (CMC) was determined by fluorescence spectroscopy with a pyrene probe. The ratio of the intensity of the peak at $337 \mathrm{~nm}$ to that at $333.5 \mathrm{~nm}$ is plotted against the log of polymer concentration and the concentration corresponding to the intersection of the two tangential lines is the CMC value (ESI: Fig. S1C $\dagger$ ). The CMCs of PU-SS-COOH and PU-SS-COOH-NH $H_{2}$ were determined to be $1.66 \times 10^{-3} \mathrm{mg} \mathrm{ml}^{-1}$ and $7.7 \times 10^{-4} \mathrm{mg} \mathrm{ml}^{-1}$, respectively. The CMC of PU-SS$\mathrm{COOH}-\mathrm{NH}_{2}$ is much lower than that of PU-SS-COOH, suggesting PU-SS-COOH- $\mathrm{NH}_{2}$ micelles are more stable than PU-SS-COOH micelles. ${ }^{38}$ Kim et al. ${ }^{39}$ lowered the CMC of PEG-PTMC block polymers by incorporating strong H-bonding urea-functional groups on the side chain of the block polymers, which
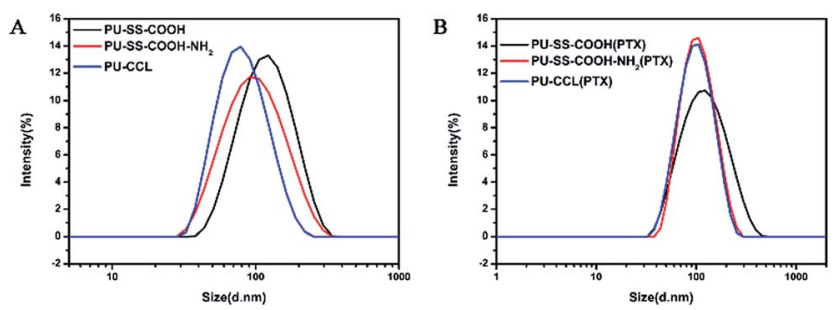

Fig. 3 Size distribution of the PU-SS-COOH, PU-SS-COOH-NH PU-CCL micelles (A) and their PTX loaded micelles (B).

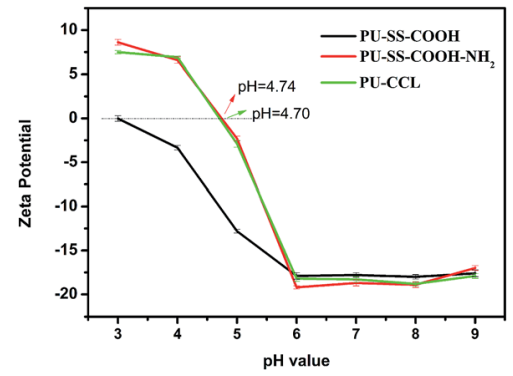

Fig. 4 Relationship of $\mathrm{pH}$ and zeta potential of PU-SS-COOH, PU-SS$\mathrm{COOH}-\mathrm{NH}_{2}$ and PU-CCL micelles.

resulted in more kinetically stable block polymer micelles. The lower CMC of PU-SS-COOH-NH $\mathrm{N}_{2}$ is, therefore, attributed to an enhanced hydrogen bonding interaction between the pendant amine groups of PU-SS-COOH- $\mathrm{NH}_{2}$.

Size and zeta potentials of the PU-SS-COOH, PU-SS-COOH$\mathrm{NH}_{2}$ and PU-CCL micelles were determined by dynamic light scattering (DLS) at $25{ }^{\circ} \mathrm{C}$ using a Zetasizer analyzer (Malvern ZetasizerNano, Zen 3690+MPT2, Malvern, UK). As presented in Table 1, the hydrodynamic diameters of the PU-SS-COOH, PUSS-COOH- $\mathrm{NH}_{2}$ and PU-CCL micelles determined by DLS are $104.93 \mathrm{~nm}, 87.02 \mathrm{~nm}$ and $77.27 \mathrm{~nm}$, respectively, with a unimodal size distribution (Fig. 3A). Notably, the particle sizes of PUSS-COOH-NH ${ }_{2}$ and PU-CCL micelles are reduced by $c a .18$ and $28 \mathrm{~nm}$, respectively, compared with that of PU-SS-COOH micelles, indicating the successful amidation of PU-SS-COOH and core-crosslinking of PU-SS-COOH- $\mathrm{NH}_{2}$ micelles. As reported in the literature, ${ }^{40}$ the particle size of crosslinked polymer micelles is usually smaller that of than their uncrosslinked micelles due to the more compact chain package of crosslinked micelles. In addition, the size reduction of PU-SS-COOH- $\mathrm{NH}_{2}$ micelles compared with PU-SS-COOH micelles may be ascribed to the enhanced interchain $\mathrm{H}$-bonding among the urethane groups and the amide groups of PU-SS-COOH- $\mathrm{NH}_{2}{ }^{40}$ After loading the drugs, the three kinds of micelles grew larger due to the encapsulation of the hydrophobic drugs into the core of the micelles (Fig. 3B). Notably, a negative surface charge at pH 7.4 was found for all three kinds of PU micelles, as shown in Table 1 , suggesting a long circulation time of these micelles in the blood.

The change of surface charge with solution $\mathrm{pH}$ was monitored by the relationship of zeta potential and $\mathrm{pH}$ value (Fig. 4). The existence of carboxyl groups and amine groups on PU-SS-

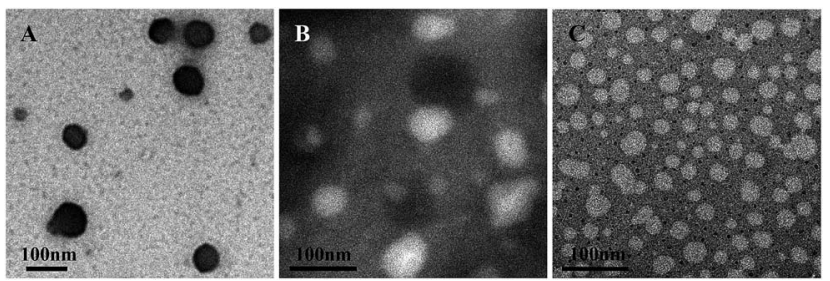

Fig. 5 TEM micrographs of PU-SS-COOH (A), PU-SS-COOH- $\mathrm{NH}_{2}$ (B) and PU-CCL (C) micelles. 

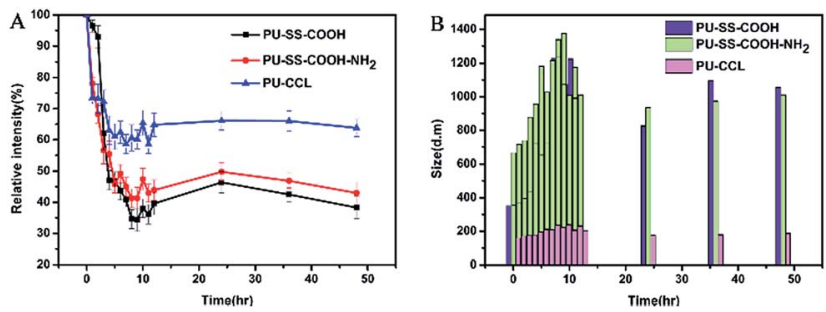

Fig. 6 DLS light intensity (A) and size change (B) of PU micelles in the presence of SDS.

$\mathrm{COOH}-\mathrm{NH}_{2}$ and PU-CCL rendered the surface charge of these two kinds of PU micelles pH-responsive characteristics. The zeta potential of all three kinds of PU micelles remained constant at $c a$. $-18 \mathrm{mV}$ when the solution's $\mathrm{pH}$ value stayed at above 6 due to the deprotonation of the carboxyl groups in these PU materials. The negative-charged PU micelles will have good protein resistance and long blood stream circulation times. ${ }^{35}$ However, zeta potential values of all the polyurethane micelles increased with a decrease of the solution's pH value when the solution was in an acidic environment $(\mathrm{pH}<6)$ as a result of the protonation of the primary amine groups and the carboxyl groups. Interestingly, as shown in Fig. 4, the surface charge of the PU-SS-COOH-NH $\mathrm{H}_{2}$ and PU-CCL micelles reversed to positive at $\mathrm{pH} 4.74$ and 4.70 , respectively, due to the protonation of the amine, which resulted in an enhanced cell uptake and the lysosome escape of these two PU micelles.

TEM micrographs of the PU micelles demonstrated that the assembled PU micelles had a spherical morphology with an average diameter of $\sim 63, \sim 50$ and $\sim 44$ nanometers for PU-SS$\mathrm{COOH}, \mathrm{PU}-\mathrm{SS}-\mathrm{COOH}-\mathrm{NH}_{2}$ and PU-CCL, respectively (Fig. 5AC). The difference in micelle sizes between DLS and TEM can be attributed to the dehydrated state of the micelles.

The micelle kinetic stability was studied by dynamic light scattering (DLS) in the presence of sodium dodecyl sulfate (SDS) known as a destabilizing agent. ${ }^{39}$ As is shown in Fig. 6A, a significant decrease in the scattered light intensity was observed in the first $10 \mathrm{~h}$ for SDS-treated PU-SS-COOH and PU$\mathrm{SS}-\mathrm{COOH}-\mathrm{NH}_{2}$ micelles, and the relative intensity was then

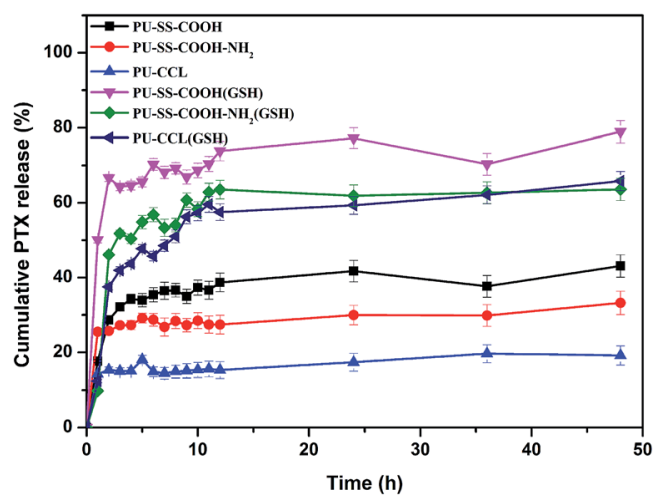

Fig. 7 Time dependent cumulative release of PTX from PU-SS$\mathrm{COOH}, \mathrm{PU}-\mathrm{SS}-\mathrm{COOH}-\mathrm{NH}_{2}$ and $\mathrm{PU}-\mathrm{CCL}$ micelles in PBS buffer solutions ( $\mathrm{pH} 7.4,10 \mathrm{mM}$ ) with and without $10 \mathrm{mM}$ of $\mathrm{GSH}$ at $37^{\circ} \mathrm{C}$. maintained at $40-45 \%$ of the original value. This suggests the dissociation of a large percentage of the micelles. In contrast, only a 35\% drop in scattered light intensity was observed for PUCCL micelles in $48 \mathrm{~h}$, indicating an enhanced stability of PUCCL micelles. Fig. 6B shows the size change of the PU micelles after treatment with SDS as a function of time. Notably, the size of the PU-CCL micelles after treatment with SDS remained stable with an average diameter less than $200 \mathrm{~nm}$. However, the sizes of the PU-SS-COOH and PU-SS-COOH-NH micelles grew instantly to about $1000 \mathrm{~nm}$ or more after treatment with SDS, suggesting a low stability of these two kinds of PU micelles.

\section{Drug loading and release}

PTX was used as a model hydrophobic drug to investigate the loading capacity of the redox active polyurethane micelles. A micelle extraction technique was used to load the PTX into polyurethane micelles. The excess PTX was removed by filtration through a $0.45 \mu \mathrm{m}$ filter. The loading level of PTX for PTXloaded micelles was determined using HPLC. The drug loading content (LC) and encapsulation efficiency for PTX loaded micelles are shown in Table 1.

The drug release behavior experiments of PTX-loaded polyurethane nanoparticles were conducted in PBS buffer solutions (pH 7.4, $10 \mathrm{mM}$ ) with and without $10 \mathrm{mM}$ of GSH at $37{ }^{\circ} \mathrm{C}$. The accumulative drug release profiles as a function of time are plotted in Fig. 7. Without the presence of GSH, an obvious initial burst release was observed for PTX-loaded PU-SS-COOH and PU-SS-COOH-NH$H_{2}$ micelles, while this was markedly

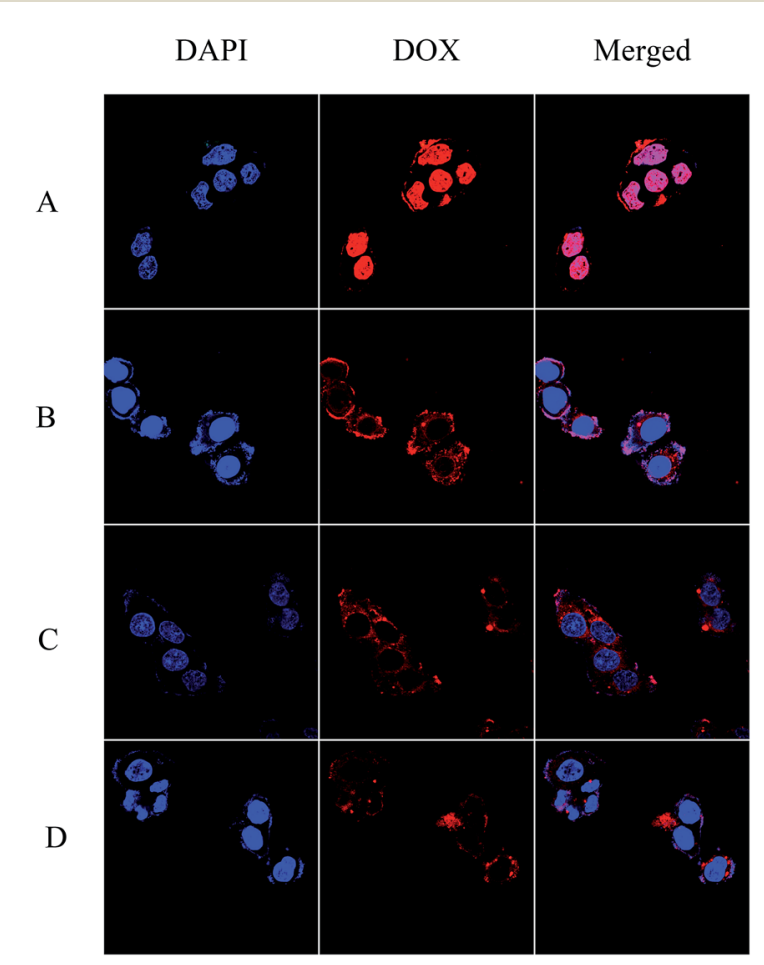

Fig. 8 CLSM images of HepG2 cells incubated with free DOX (A), and DOX loaded PU-SS-COOH (B), PU-SS-COOH-NH (D) micelles for $2 \mathrm{~h}$. Scale bar $=10 \mu \mathrm{m}$. 

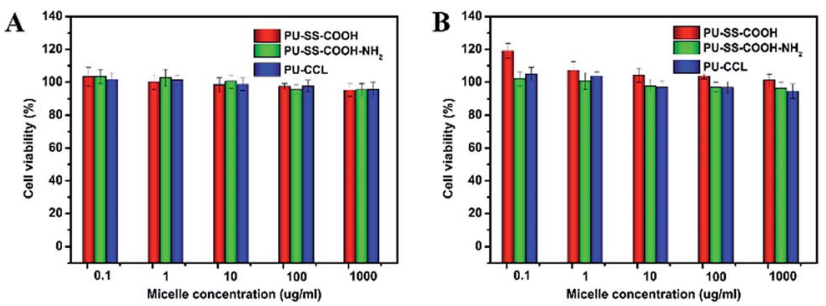

Fig. 9 Viability of HUVECs (A) and HepG2 cells (B) after $48 \mathrm{~h}$ of incubation with various concentrations of empty reduction-sensitive polyurethane micelles determined by the CCK8 assay.

suppressed for drug loaded PU-CCL micelles. For instance, only $19 \%$ of PTX was released from drug encapsulated PU-CCL micelles at $48 \mathrm{~h}$ without the presence of GSH (Fig. 7), and $43 \%$ and $33 \%$ were released from PTX encapsulated uncrosslinked PU-SS-COOH and PU-SS-COOH- $\mathrm{NH}_{2}$ at $48 \mathrm{~h}$. This indicated a better stability of PTX loaded PU-CCL micelles in PBS than that of the other two drug loaded uncross-linked PU micelles. In addition, drug loaded PU-SS-COOH- $\mathrm{NH}_{2}$ micelles show an enhanced stability over PTX loaded PU-SS-COOH micelles, which is consistent with the CMC results. However, PTX release from all the three PU micelles was enhanced dramatically when $10 \mathrm{mM}$ GSH was present in the PBS solution, causing $78 \%, 62 \%$ and $60 \%$ to be released from PU-SS-COOH, PU-SS-COOH-NH $\mathrm{H}_{2}$ and PU-CCL micelles, respectively, in $48 \mathrm{~h}$. The enhanced drug release in the presence of GSH arose from the disassembly of polyurethane micelles due to the GSHinduced disulfide cleavage.

\section{Internalization and intracellular release of the polyurethane micelle payload}

The cellular uptake and intracellular drug release behavior of DOX-loaded nanoparticles in HepG2 cells were analyzed by confocal laser scanning microscopy (CLSM). Since PTX molecules are not fluorescent, doxorubicin (DOX, red fluorescence) was encapsulated in hydrophobic micellar cores using the dialysis method to label the nanocarriers and to track the internalization and intracellular localization of DOX (red fluorescence) in HepG2 cells. $^{40}$ The nuclei of HepG2 cells were stained by DAPI, which
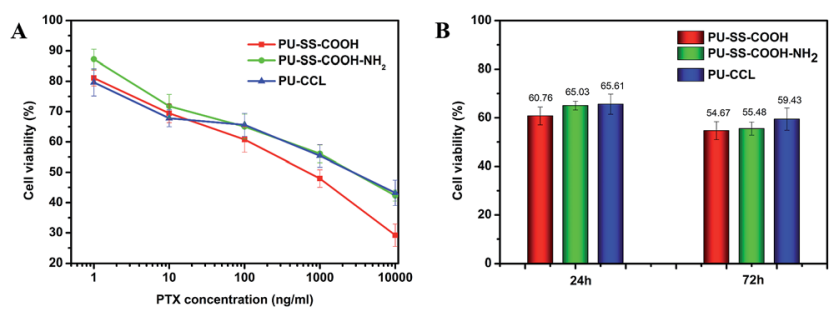

Fig. 10 Cytotoxicity of PTX-loaded polyurethane micelles against HepG2 cells after incubation for $24 \mathrm{~h}$ (A). The anti-tumor activity of PTXloaded PU-CCL micelles in HepG2 cells. PTX-loaded PU-SS-COOH and $\mathrm{PU}-\mathrm{SS}-\mathrm{COOH}-\mathrm{NH}_{2}$ were used as controls. PTX dosage was $100.0 \mathrm{ng}$ $\mathrm{ml}^{-1}$. The cells were incubated with micellar PTX for 24 or $72 \mathrm{~h}$. Data are presented as the average \pm standard deviation $(n=4)(B)$. presented blue fluorescence to distinguish it from the red fluorescence. Fig. 8 shows the CLSM images of HepG2 cells incubated with DOX-loaded polyurethane micelles for 2 h. As shown in Fig. 8, after $2 \mathrm{~h}$ of cell incubation, a strong red fluorescence of free DOX was observed in nuclei, which even turned pink in the merged fluorescence images as a result of the overlapping fluorescence of DAPI and DOX. DOX fluorescence was observed in the cytoplasm and nuclei of DOX loaded PU-SS-COOH-NH PU-CCL micelles, which suggests that DOX-loaded polyurethane micelles were internalized and DOX was released to reach the cell nuclei. ${ }^{41,42}$ In addition, DOX loaded PU-CCL showed a weaker fluorescence intensity as compared to that of drug loaded noncrosslinked micelles due to the enhanced stabilities of PU-CCL. ${ }^{26}$

\section{In vitro cytotoxicities of polyurethane micelles and PTX- loaded polyurethane micelles}

The in vitro cytotoxicity of drug-free and drug-loaded polyurethane micelles was evaluated in the CCK8 assay. Fig. 9 shows the effect of PU-SS-COOH, PU-SS-COOH-NH concentration on the viability of HUVECs and HepG2 cells. The results demonstrate that empty PU-SS-COOH, PU-SS-COOH-NH and PU-CCL micelles show very low cytotoxicity (greater than $90 \%$ cell viability) in two different cell lines even at micelle concentrations up to $1 \mathrm{mg} \mathrm{ml} \mathrm{m}^{-1}$; suggesting the nontoxic nature of polyurethane micelles to HUVECs and HepG2 cells.

Further, the in vitro cytotoxicity of PTX-loaded PU-SS-COOH, PU-SS-COOH-NH $\mathrm{H}_{2}$ and PU-CCL micelles is shown in Fig. 10. Fig. 10A shows the cytotoxicity results given as a function of PTX concentration from 1 to $10000 \mathrm{ng} \mathrm{ml}^{-1}$. As the test concentration was increased to $100 \mathrm{ng} \mathrm{m}{ }^{-1}$, PU-CCL encapsulated PTX exhibited a higher cell viability $(65.61 \%)$ than PU-SS-COOH and PU-SS-COOH- $\mathrm{NH}_{2}$ encapsulated PTX. The $\mathrm{IC}_{50}$ (i.e., inhibitory concentration to produce $50 \%$ cell death) values of PU-SS$\mathrm{COOH}, \mathrm{PU}-\mathrm{SS}-\mathrm{COOH}-\mathrm{NH}_{2}$ and PU-CCL encapsulated PTX were determined to be $\sim 357, \sim 2239$ and $\sim 2655 \mathrm{ng} \mathrm{ml}^{-1}$, respectively, for HepG2 cells $(P<0.05)$. The results reveal that the PU-CCL micelles provide a less efficient intracellular delivery of PTX as compared to PU-SS-COOH micelles. The reason is that the PUCCL micelles were more stable than PU-SS-COOH, which leads to a less efficient intracellular delivery. HepG2 cells were treated for $24 \mathrm{~h}$ or $72 \mathrm{~h}$ with PTX-loaded micelles (PTX dosage: $100 \mathrm{ng} \mathrm{ml}^{-1}$ ) as shown in Fig. 10B. Interestingly, cell viabilities of $65.6 \%$ and $59.4 \%(P<0.05)$ were observed for HepG2 cells following incubation with PTX-loaded crosslinked micelles for 24 and $72 \mathrm{~h}$, respectively, which are analogous to those obtained for PTX-loaded non-crosslinked controls (cell viabilities of $65 \%$ and $55.5 \%(P<0.05)$ for 24 and $72 \mathrm{~h}$ incubation, respectively). The cell viability declined with an extending time of cultivation. This phenomenon shows a drug sustained release.

\section{Conclusions}

In summary, novel core cross-linked polyurethane micelles with redox sensitive and $\mathrm{pH}$-responsive surface charge switchable properties were prepared and investigated as anti-cancer drug carriers. As a starting material, amphiphilic multi-blocked 
polyurethane with pendant carboxyl groups and disulfides in the hard segment (PU-SS-COOH) was synthesized firstly. The PU-SS$\mathrm{COOH}$ was further functionalized by 1,6-diaminohexane, which resulted in polyurethane with pendant amine and carboxyl groups (PU-SS-COOH-NH$H_{2}$ ). Then the PU-SS-COOH-NH $\mathrm{H}_{2}$ was assembled into micelles and hexamethylene-1,6-diisocyanate (HDI) was reacted with the amine groups which resulted in core crosslinked PU micelles (PU-CCL). All the three kinds of PU micelles released their payloads in the presence of GSH, while PU micelles with both amine and carboxyl groups, i.e. PU-SS-COOH$\mathrm{NH}_{2}$ and PU-CCL micelles, showed $\mathrm{pH}$-responsive charge switchable properties. Furthermore, the stability of the core crosslinked PU micelles (PU-CCL) enhanced dramatically while the premature release decreased compared with non-crosslinked micelles. The in vitro cytotoxicity and cell uptake of the PTX (or DOX, used to illustrate the cell uptake) loaded micelles was also assessed in HepG2 cells. The cross-linked reduction responsive biodegradable micelles, having superior extracellular stability and providing rapid intracellular drug release, may hold great potential as a bio-triggered drug delivery system for cancer therapy.

\section{Acknowledgements}

This work was financially supported by Applied Basic Research Programs Foundation of Sichuan Province (No. 2015JY0126) and the Innovation Scientific Research Program for Graduates in Southwest University for Nationalities (CX2015SZ056).

\section{Notes and references}

1 R. Savic, L. Luo, A. Eisenberg and D. Maysinger, Science, 2003, 300, 615.

2 N. Ma, Y. Li, H. Xu, Z. Wang and X. Zhang, J. Am. Chem. Soc., 2010, 132, 442.

3 M. Ding, J. Li, X. He, N. Song, H. Tan, Y. Zhang, L. Zhou, Q. Gu, H. Deng and Q. Fu, Adv. Mater., 2012, 24, 3639.

4 S. Yu, C. He, J. Ding, Y. Cheng, W. Song, X. Zhuang and X. Chen, Soft Matter, 2013, 9, 2637.

5 D. Xu, Y. Su, L. Zhao, F. Meng, C. Liu, Y. Guan, J. Zhang and J. Luo, J. Biomed. Mater. Res., Part A, 2017, 105(2), 531.

6 Y. Matsumura and H. Maeda, Cancer Res., 1986, 46, 6387.

7 M. Talelli, M. Barz, C. J. F. Rijcken, F. Kiessling, W. E. Hennink and T. Lammers, Nano Today, 2015, 10, 93.

8 H. Maeda, H. Nakamura and J. Fang, Adv. Drug Delivery Rev., 2013, 65, 71.

9 H. Maeda, J. Controlled Release, 2012, 164, 138.

10 R. K. Jain and T. Stylianopoulos, Nat. Rev. Clin. Oncol, 2010, 7, 653.

11 Q. Sun, X. Sun, X. Ma, Z. Zhou, E. Jin, B. Zhang, Y. Shen, E. A. Van Kirk, W. J. Murdoch, J. R. Lott, T. P. Lodge, M. Radosz and Y. Zhao, Adv. Mater., 2014, 01, 554.

12 Q. Zhang, Na R. Ko and J. K. Oh, Chem. Commun., 2012, 48, 7542.

13 E. Fleige, M. A. Quadir and R. Haag, Adv. Drug Delivery Rev., 2012, 64, 866.
14 F. Meng, R. Cheng, C. Deng and Z. Zhong, Mater. Today, 2012, 15, 436.

15 W. Chen, P. Zhong, F. Meng, R. Cheng, C. Deng, J. Feijen and Z. Zhong, J. Controlled Release, 2013, 169, 171.

16 S. Mura, J. Nicolas and P. Couvreur, Nat. Mater., 2013, 12, 991. 17 X. He, M. Ding, J. Li, H. Tan, Q. Fu and L. Li, RSC Adv., 2014, 4, 24736.

18 Y. Yao, H. Xu, C. Liu, Y. Guan, D. Xu, J. Zhang, Y. Su, L. Zhao and J. Luo, RSC Adv., 2016, 6, 9082.

19 S. Yu, J. Ding, C. He, Y. Cao, W. Xu and X. Chen, Adv. Healthcare Mater, 2014, 3, 752.

20 Y. Li, K. Xiao, W. Zhu, W. Deng and K. S. Lam, Adv. Drug Delivery Rev., 2014, 66, 58.

21 W. Chen, F. Meng, R. Cheng, C. Deng, J. Feijen and Z. Zhong, J. Controlled Release, 2015, 210, 125.

22 H. S. Han, Ki Y. Choi, H. Ko, J. Jeon, G. Saravanakumar, Y. D. Suh, D. S. Lee and J. H. Park, J. Controlled Release, 2015, 200, 158.

23 R. K. O'Reilly, C. J. Hawker and K. L. Wooley, Chem. Soc. Rev., 2006, 35, 1068.

24 E. S. Read and S. P. Armes, Chem. Commun., 2007, 3021.

25 Y.-L. Li, L. Zhu, Z. Liu, R. Cheng, F. Meng, J.-H. Cui, S.-J. Ji and Z. Zhong, Angew. Chem., Int. Ed., 2009, 48, 9914.

26 J. Dai, S. Lin, D. U. Cheng, S. Zou and X. Shuai, Angew. Chem., Int. Ed., 2011, 50, 9404.

27 A. Na Koo, H. Jae Lee, S. Eun Kim, J. H. Chang, C. Park, C. Kim, J. H. Park and S. C. Lee, Chem. Commun., 2008, 6570.

28 R. Sun, Q. Luo, C. Gao, Y. Wang, L. Gao, H. Du, Y. Huang, X. Li, Z. Shen and W. Zhu, Polym. Chem., 2014, 5, 4879.

29 G. Kwon, S. Suwa, M. Yokoyama, T. Okano, Y. Sakurai and K. Kataoka, J. Controlled Release, 1994, 29, 17.

30 H. Liu, S. Farrell and K. Uhrich, J. Controlled Release, 2000, 68, 167.

31 R. Savic, A. Eisenberg and D. Maysinger, J. Drug Targeting, 2006, 14, 343.

32 Y. Yao, D. Xu, C. Liu, Y. Guan, J. Zhang, Y. Su, L. Zhao, F. Meng and J. Luo, RSC Adv., 2016, 6, 97684.

33 J.-W. Yoo, N. Doshi and S. Mitragotri, Adv. Drug Delivery Rev., 2011, 63, 1247.

34 V. Mailander and K. Landfester, Biomacromolecules, 2009, 10(9), 2379.

35 Y. Huang, Z. Tang, X. Zhang, H. Yu, H. Sun, X. Pang and X. Chen, Biomacromolecules, 2013, 14, 2023.

36 L. Wu, L. Zhang, G. Shi and C. Ni, Mater. Sci. Eng., C, 2016, 61, 278.

37 Y. Tao, R. Liu, M. Chen, C. Yang and X. Liu, J. Mater. Chem., 2012, 22, 373.

38 J. Liu, F. Zeng and C. Allen, Eur. J. Pharm. Biopharm., 2007, 65, 309.

39 S. H. Kim, J. P. K. Tan, F. Nederberg, K. Fukushima, J. Colson, C. Yang, A. Nelson, Y.-Y. Yang and J. L. Hedrick, Biomaterials, 2010, 31, 8063.

40 Y. Wu, W. Chen, F. Meng, Z. Wang, R. Cheng, C. Deng, H. Liu and Z. Zhong, J. Controlled Release, 2012, 164, 338.

41 Z. Ge and S. Liu, Chem. Soc. Rev., 2013, 42, 7289.

42 P. S. Pramod, R. Shah and M. Jayakannan, Nanoscale, 2015, 7, 6636. 